\title{
Convergence Analysis of Parallel S-Iteration Process for System of Generalized Variational Inequalities
}

\author{
D. R. Sahu, ${ }^{1}$ Shin Min Kang, ${ }^{2,3}$ and Ajeet Kumar ${ }^{1}$ \\ ${ }^{1}$ Department of Mathematics, Institute of Science, Banaras Hindu University, Varanasi 221005, India \\ ${ }^{2}$ Department of Mathematics and the RINS, Gyeongsang National University, Jinju 52828, Republic of Korea \\ ${ }^{3}$ Center for General Education, China Medical University, Taichung 40402, Taiwan
}

Correspondence should be addressed to Shin Min Kang; smkang@gnu.ac.kr

Received 8 July 2017; Accepted 27 August 2017; Published 12 October 2017

Academic Editor: Tomonari Suzuki

Copyright (C) 2017 D. R. Sahu et al. This is an open access article distributed under the Creative Commons Attribution License, which permits unrestricted use, distribution, and reproduction in any medium, provided the original work is properly cited.

\begin{abstract}
We consider a new system of generalized variational inequalities (SGVI) defined on two closed convex subsets of a real Hilbert space. To find the solution of considered SGVI, a parallel Mann iteration process and a parallel S-iteration process have been proposed and the strong convergence of the sequences generated by these parallel iteration processes is discussed. Numerical example illustrates that the proposed parallel $S$-iteration process has an advantage over parallel Mann iteration process in computing altering points of some mappings.
\end{abstract}

\section{Introduction}

Variational inequalities are the most interesting and important mathematical problems and have been studied intensively in the past years. The variational inequality problem was first introduced and studied by Stampacchia [1] in 1964, which is defined as follows.

Let $C$ be a nonempty closed convex subset of a real Hilbert space $H$ and let $T: C \rightarrow H$ be a nonlinear mapping. Then the classical variational inequality problem is to find a point $x^{*} \in C$ such that

$$
\left\langle T x^{*}, x-x^{*}\right\rangle \geq 0 \quad \forall x \in C .
$$

The problem (1) is denoted by $V I(C, T)$ and the set of solutions of (1) is denoted and defined by $\Omega[V I(C, T)]=$ $\left\{x^{*} \in C:\left\langle T x^{*}, x-x^{*}\right\rangle \geq 0\right.$ for all $\left.x \in C\right\}$. We denote by $\operatorname{Fix}(T)$ the set of fixed points of $T$. It is well known that the variational inequality problem (1) is equivalent to the following fixed point problem:

$$
\text { find } x^{*} \in C \text { such that } x^{*}=P_{C}(I-\mu T) x^{*} \text {, }
$$

where $P_{C}$ is the metric projection from $H$ onto $C, \mu>0$ is a constant, and $I$ is the identity mapping from $H$ into itself. It is well known that if the mapping $T$ is $k$-Lipschitzian and $\eta$-strongly monotone, then the operator $P_{C}(I-\mu T)$ is a contraction on $C$ provided that $0<\mu<2 \eta / k^{2}$. In this case, the Banach contraction principle guarantees that $\operatorname{VI}(C, T)$ has a unique solution $x^{*}$ and the sequence of Picard iteration method given by

$$
x_{n+1}=P_{C}(I-\mu T) x_{n} \quad \forall n \in \mathbb{N}
$$

converges strongly to $x^{*}$. This method is called the projected gradient method [2]. This method has been widely used in many practical problems, due partially to its fast convergence.

In 2007, Agarwal et al. [3] posed the following query.

Question 1. Is it possible to develop an iterative method whose rate of convergence is faster than the Picard iteration method for contraction mappings?

They introduced the following iteration process known as $S$-iteration process as an answer to Question 1: let $C$ be a nonempty convex subset of a normed linear space $X$, and let 
$T: C \rightarrow C$ be an operator. Then, for arbitrary $x_{1} \in C$, the $S$-iteration process is defined by

$$
\begin{aligned}
x_{n+1} & =\left(1-\alpha_{n}\right) T x_{n}+\alpha_{n} T y_{n}, \\
y_{n} & =\left(1-\beta_{n}\right) x_{n}+\beta_{n} T x_{n} \quad \forall n \in \mathbb{N},
\end{aligned}
$$

where $\left\{\alpha_{n}\right\}$ and $\left\{\beta_{n}\right\}$ are real sequences in $(0,1)$ satisfying some suitable conditions. In [4], Sahu proved that the rate of convergence of $S$-iteration process for contraction mappings is faster than that of Picard [5] and Mann [6] iteration processes by providing a numerical example. The $S$-iteration process is more applicable than the Picard [5], Mann [6], and Ishikawa [7] iteration processes because it converges faster than these iteration processes for contraction mappings and also works for nonexpansive mappings. Due to the super rate of convergence of above iteration process, Agarwal et al. [3] called it the $S$-iteration process. Due to its fastness, in recent years, the $S$-iteration process attracted many researchers as an alternate iteration process and is used for solving fixed point problems, common fixed point problems, convex minimization problems, the problem of solving nonlinear operator equations, and other allied areas (see [8-10]). Moreover, the idea of $S$-iteration process is applied by Cholamjiak et al. [11] for finding a minimizer of a convex function and fixed points of nonexpansive mappings in CAT(0) space setting. Sahu [4] also introduced the notion of $S$-operator of a mapping $T$ generated by $\alpha \in(0,1)$ and $T$ and normal $S$-iteration process in the following way: let $C$ be a nonempty convex subset of a normed linear space $X$ and let $T: C \rightarrow C$ be an operator. Then, for arbitrary $x_{1} \in C$, the normal $S$-iteration process is defined by

$$
x_{n+1}=T\left[\left(1-\alpha_{n}\right) x_{n}+\alpha_{n} T\left(x_{n}\right)\right] \quad \forall n \in \mathbb{N},
$$

where $\left\{\alpha_{n}\right\}$ is a sequence of real numbers in $(0,1)$. In 2017, Verma and Shukla [12] designed some new algorithms based on $S$-iteration processes and named them as $S$-iteration-based forward-backward algorithm (SFBA) and normal $S$-iterationbased forward-backward algorithm (NSFBA) and performed the nice experiments of the high-dimensional real datasets for SFBA, NSFBA, and others.

On the other hand, in Hilbert spaces, projection type methods have played a very crucial role in the numerical resolution of variational inequalities depending on their convergence analysis. By virtue of the projection, in 2011, Ceng et al. [13] proposed the following iterative method:

$$
x_{n+1}=P_{C}\left[\alpha_{n} \gamma V x_{n}+\left(I-\alpha_{n} \mu F\right) T x_{n}\right] \quad \forall n \in \mathbb{N},
$$

where $F: C \rightarrow H$ is $k$-Lipschitzian and $\eta$-strongly monotone operator with $k>0, \eta>0, V: C \rightarrow H$ is an $L$-Lipschitzian mapping with $L \geq 0, T: C \rightarrow C$ is a nonexpansive mapping with $\operatorname{Fix}(T) \neq \emptyset,\left\{\alpha_{n}\right\} \subset(0,1)$, and $x_{1} \in C$ an arbitrary initial point. They proved that the sequence $\left\{x_{n}\right\}$ generated by the iterative method (6) converges strongly to a fixed point $x^{*}$ of $T$ which solves the following variational inequality problem:

$$
\left\langle(\mu F-\gamma V) x^{*}, x-x^{*}\right\rangle \geq 0 \quad \forall x \in C:=\operatorname{Fix}(T) .
$$

In 2001, Verma [14] generalized the concept of variational inequalities to a system of nonlinear variational inequalities (SNVI) in the following way: find $x^{*}, y^{*} \in C$ such that

$$
\begin{array}{ll}
\left\langle\rho T\left(y^{*}\right)+x^{*}-y^{*}, x-x^{*}\right\rangle \geq 0, & \forall x \in C, \\
\left\langle\eta T\left(x^{*}\right)+y^{*}-x^{*}, x-y^{*}\right\rangle \geq 0, & \forall x \in C,
\end{array}
$$

where $T: C \rightarrow H$ is any mapping and $\eta>0$ and $\rho>0$ are constants. To solve (8), he introduced the following iterative method:

$$
\begin{aligned}
y_{n} & =P_{C}\left[x_{n}-\eta T\left(x_{n}\right)\right], \\
x_{n+1} & =\left(1-\alpha_{n}\right) x_{n}+\alpha_{n} P_{C}\left[y_{n}-\rho T\left(y_{n}\right)\right],
\end{aligned}
$$

and he proved that the sequences $\left\{x_{n}\right\}$ and $\left\{y_{n}\right\}$ generated by (9) converge to $x^{*}$ and $y^{*}$, respectively. In 2005, Verma [15] also introduced the general model for two-step projection methods for applying the approximation solvability of SNVI in Hilbert space setting as follows: let $C$ be a nonempty closed convex subset of a real Hilbert space $H$ and let $T: C \rightarrow H$ be a nonlinear mapping. For arbitrary chosen initial point $x_{1} \in C$, let $\left\{x_{n}\right\}$ and $\left\{y_{n}\right\}$ be the sequences in $C$ defined by

$$
\begin{aligned}
y_{n} & =\left(1-\beta_{n}\right) x_{n}+\beta_{n} P_{C}\left[x_{n}-\eta T\left(x_{n}\right)\right], \\
x_{n+1} & =\left(1-\alpha_{n}\right) x_{n}+\alpha_{n} P_{C}\left[y_{n}-\rho T\left(y_{n}\right)\right],
\end{aligned}
$$

where $\eta>0, \rho>0$ and $0 \leq \alpha_{n}, \beta_{n} \leq 1$. Further, problem (8) is equivalent to the following projection formulas:

$$
\begin{aligned}
& x^{*}=P_{C}(I-\rho T) y^{*}, \\
& y^{*}=P_{C}(I-\eta T) x^{*},
\end{aligned}
$$

for a monotone mapping $T: C \rightarrow H$. The problem of finding the solutions of (11) by using iterative methods has been studied by many authors (see [15-22]). A more general case has been studied in [23].

Parallel iteration processes have their own advantages. A variety of problems have been dealt with in these iteration processes (see $[24,25]$ and the references therein). Recently, Sahu [26] introduced the notion of altering points of nonlinear mappings and following the idea of $S$-operator and normal $S$-iteration process, he [26] introduced a parallel $S$-iteration process for finding altering points of nonlinear mappings as follows.

Let $C_{1}$ and $C_{2}$ be two nonempty closed convex subsets of a Banach space $X$ and let $T_{1}: C_{1} \rightarrow C_{2}$ and $T_{2}: C_{2} \rightarrow C_{1}$ be two mappings. Then, for $\alpha \in(0,1)$ and arbitrary $\left(x_{1}, y_{1}\right) \in$ $C_{1} \times C_{2}$, the parallel normal $S$-iteration process is defined by

$$
\begin{aligned}
& x_{n+1}=T_{2}\left[(1-\alpha) y_{n}+\alpha T_{1}\left(x_{n}\right)\right], \\
& y_{n+1}=T_{1}\left[(1-\alpha) x_{n}+\alpha T_{2}\left(y_{n}\right)\right] \quad \forall n \in \mathbb{N} .
\end{aligned}
$$

The following convergence result is given in [26].

Theorem 1 (see [26]). Let $C_{1}$ and $C_{2}$ be two nonempty closed convex subsets of a Banach space $X$. Let $T_{1}: C_{1} \rightarrow C_{2}$ and $T_{2}$ : $C_{2} \rightarrow C_{1}$ be two Lipschitz continuous mappings with Lipschitz 
constants $k_{1}<1$ and $k_{2}<1$, respectively. Then the sequence $\left\{\left(x_{n}, y_{n}\right)\right\}$ in $C_{1} \times C_{2}$ generated by the parallel $S$-iteration process (12) converges strongly to a unique point $\left(x^{*}, y^{*}\right) \in C_{1} \times C_{2}$ such that $x^{*}$ and $y^{*}$ are altering points of mappings $T_{1}$ and $T_{2}$.

In this paper, motivated by the work of Ceng et al. [13], Verma [14, 15], Hao et al. [23], and Sahu [26], we consider a new SGVI defined on two closed convex subsets of a real Hilbert space and propose a parallel Mann and a more general parallel $S$-iteration process for solving considered SGVI in the context of altering points and study the strong convergence of the sequences generated by the proposed algorithms to altering points of some nonlinear mappings.

\section{Preliminaries}

Throughout this paper, the symbol $\mathbb{N}$ stands for the set of all natural numbers.

Let $C$ be a nonempty subset of a real Hilbert space $H$ with inner product $\langle\cdot, \cdot\rangle$ and norm $\|\cdot\|$, respectively. A mapping $T: C \rightarrow H$ is called

(1) monotone if

$$
\langle T x-T y, x-y\rangle \geq 0 \quad \forall x, y \in C,
$$

(2) $\eta$-strongly monotone if there exists a positive real number $\eta$ such that

$$
\langle T x-T y, x-y\rangle \geq \eta\|x-y\|^{2} \quad \forall x, y \in C,
$$

(3) $k$-Lipschitzian if there exists a constant $k \geq 0$ such that

$$
\|T x-T y\| \leq k\|x-y\| \quad \forall x, y \in C,
$$

(4) $\theta$-contraction if there exists a constant $\theta \in[0,1)$ such that

$$
\|T x-T y\| \leq \theta\|x-y\| \quad \forall x, y \in C,
$$

(5) nonexpansive if

$$
\|T x-T y\| \leq\|x-y\| \quad \forall x, y \in C .
$$

Definition 2 (see [26]). Let $C_{1}$ and $C_{2}$ be two nonempty subsets of a metric space $X$. Then $x^{*} \in C_{1}$ and $y^{*} \in C_{2}$ are altering points of mappings $T_{1}: C_{1} \rightarrow C_{2}$ and $T_{2}: C_{2} \rightarrow C_{1}$ if

$$
\begin{aligned}
& T_{1}\left(x^{*}\right)=y^{*}, \\
& T_{2}\left(y^{*}\right)=x^{*} .
\end{aligned}
$$

The set of altering points of mappings $T_{1}$ and $T_{2}$ is denoted and defined by

$$
\begin{aligned}
& \operatorname{Alt}\left(T_{1}, T_{2}\right)=\left\{\left(x^{*}, y^{*}\right) \in C_{1} \times C_{2}: T_{1}\left(x^{*}\right)\right. \\
& \left.=y^{*}, T_{2}\left(y^{*}\right)=x^{*}\right\} .
\end{aligned}
$$

We now give some numerical examples in support of the definition of altering points of some nonlinear mappings as follows.
Example 3 (see [26]). Let $X=[0,1], C_{1}=[0,1 / 2]$, and $C_{2}=$ $[1 / 2,1]$. Define $T_{1}: C_{1} \rightarrow C_{2}$ and $T_{2}: C_{2} \rightarrow C_{1}$ by $T_{i}(x)=$ $1-x$ for $i=1,2$. Note that $T_{2} T_{1}: C_{1} \rightarrow C_{1}$ is defined by $T_{2} T_{1} x=T_{2}(1-x)=x$. Thus, each point of $C_{1}$ is a fixed point of $T_{2} T_{1}$. Then altering points $x^{*} \in C_{1}$ and $y^{*} \in C_{2}$ of $T_{1}$ and $T_{2}$ are given by the relation $x^{*}+y^{*}=1$. Indeed,

$$
\operatorname{Alt}\left(T_{1}, T_{2}\right)=\left\{\left(x^{*}, y^{*}\right) \in C_{1} \times C_{2}: x^{*}+y^{*}=1\right\} .
$$

Example 4. Let $X=\mathbb{R}^{2}, C_{1}=\left\{(x, y) \in \mathbb{R}^{2}: x \geq 0, y \geq 0\right\}$, and $C_{2}=\left\{(x, y) \in \mathbb{R}^{2}: x \leq 0, y \leq 0\right\}$. Let $T_{1}: C_{1} \rightarrow C_{2}$ and $T_{2}: C_{2} \rightarrow C_{1}$ be two mappings defined, respectively, by

$$
\begin{aligned}
& T_{1}(x, y)=(-x,-y-1) \quad \forall(x, y) \in C_{1}, \\
& T_{2}(x, y)=\left(\frac{1-x}{2}, \frac{1-y}{2}\right) \quad \forall(x, y) \in C_{2} .
\end{aligned}
$$

Note that $T_{2} T_{1}: C_{1} \rightarrow C_{1}$ is defined by $T_{2} T_{1}(x, y)=$ $T_{2}(-x,-y-1)=((x+1) / 2,(y+2) / 2)$. Clearly $(1,2) \in C_{1}$ and $(-1,-3) \in C_{2}$ are fixed points of $T_{2} T_{1}$ and $T_{1} T_{2}$, respectively. Therefore, $x^{*}=(1,2)$ and $y^{*}=(-1,-3)$ are altering points of mappings $T_{1}$ and $T_{2}$.

Let $C$ be a nonempty closed convex subset of $H$. Then, for any $x \in H$, there exists a unique nearest point $P_{C}(x)$ of $C$ such that

$$
\left\|x-P_{C}(x)\right\| \leq\|x-y\| \quad \forall y \in C .
$$

The mapping $P_{C}$ is called the metric projection [27] from $H$ onto $C$. It is remarkable that the metric projection mapping $P_{C}$ is nonexpansive from $H$ onto $C$ (see Agarwal et al. [28]).

We need the following technical lemmas.

Lemma 5 (see [28]). Let $C$ be a nonempty closed convex subset of a real Hilbert space $H$ and let $P_{C}$ be the metric projection from $H$ onto $C$. Given $x \in H$ and $z \in C$, then $z=P_{C}(x)$ if and only if $\langle x-z, z-y\rangle \geq 0$ for all $y \in C$.

Lemma 6 (see [29]). Let $C$ be a nonempty subset of a real Hilbert space $H$. Suppose that $\lambda \in(0,1)$ and $\mu>0$. Let $F: C \rightarrow H$ be a $k$-Lipschitzian and $\eta$-strongly monotone operator. Define the mapping $T_{\lambda}: C \rightarrow H$ by

$$
T_{\lambda}(x)=(I-\lambda \mu F)(x) \quad \forall x \in C, \lambda \in(0,1) .
$$

Then $T_{\lambda}$ is a contraction provided $0<\mu<2 \eta / k^{2}$. More precisely, for $\mu \in\left(0,2 \eta / k^{2}\right)$,

$$
\left\|T_{\lambda}(x)-T_{\lambda}(y)\right\| \leq(1-\lambda \tau)\|x-y\| \quad \forall x, y \in C,
$$

where $\tau=1-\sqrt{1-\mu\left(2 \eta-\mu k^{2}\right)} \in(0,1]$.

Lemma 7 (see [17]). Let $\left\{a_{n}\right\},\left\{b_{n}\right\}$, and $\left\{c_{n}\right\}$ be three nonnegative real sequences satisfying the following conditions:

$$
a_{n+1} \leq\left(1-\alpha_{n}\right) a_{n}+b_{n}+c_{n} \quad \forall n \geq n_{0},
$$

where $n_{0}$ is some nonnegative integer, $\alpha_{n} \in(0,1)$ with $\sum_{n=0}^{\infty} \alpha_{n}=\infty, b_{n}=\circ\left(\alpha_{n}\right)$, and $\sum_{n=0}^{\infty} c_{n}<\infty$. Then $\lim _{n \rightarrow \infty} a_{n}=$ 0 . 
Lemma 8 (see [26, Theorem 3.1]). Let $C_{1}$ and $C_{2}$ be two nonempty closed subsets of a complete metric space $X$. Suppose that $S_{1}: C_{1} \rightarrow C_{2}$ and $S_{2}: C_{2} \rightarrow C_{1}$ be two Lipschitz continuous mappings with Lipschitz constants $k_{1}$ and $k_{2}$, respectively, such that $k_{1} k_{2}<1$. Then the following holds:

(a) There exists a unique point $\left(x^{*}, y^{*}\right) \in C_{1} \times C_{2}$ such that $x^{*}$ and $y^{*}$ are altering points of mappings $S_{1}$ and $S_{2}$.

(b) For arbitrary $x_{1} \in C_{1}$, a sequence $\left\{\left(x_{n}, y_{n}\right)\right\} \in C_{1} \times C_{2}$ generated by

$$
\begin{aligned}
y_{n} & =S_{1} x_{n}, \\
x_{n+1} & =S_{2} y_{n} \quad \forall n \in \mathbb{N}
\end{aligned}
$$

converges to $\left(x^{*}, y^{*}\right)$.

\section{Main Results}

In this section, we introduce a new system of generalized variational inequalities and new iterative algorithms for solving the proposed system of generalized variational inequalities in the framework of real Hilbert spaces.

Let $C_{1}$ and $C_{2}$ be nonempty closed convex subsets of a real Hilbert space $H$ and let $T_{1}: C_{1} \rightarrow C_{2}$ and $T_{2}: C_{2} \rightarrow C_{1}$ be some mappings. Let $g_{1}, g_{2}: H \rightarrow H$ be mappings. Consider a general system of generalized variational inequalities (SGVI) defined on $C_{1}$ and $C_{2}$ as follows.

Find $\left(x^{*}, y^{*}\right) \in \mathrm{C}_{1} \times \mathrm{C}_{2}$ such that

$$
\begin{array}{ll}
\begin{array}{l}
\left\langle s T_{1}\left(x^{*}\right)+y^{*}-g_{1}\left(x^{*}\right), g_{1}(y)-y^{*}\right\rangle \geq 0, \\
\forall y \in C_{2},
\end{array} & \\
\left\langle t T_{2}\left(y^{*}\right)+x^{*}-g_{2}\left(y^{*}\right), g_{2}(x)-x^{*}\right\rangle \geq 0, &
\end{array}
$$

$\forall x \in C_{1}$,

where $s>0$ and $t>0$ are constants.

Remark 9. If $T_{1}=T_{2}=T, g_{1}=g_{2}=I$, and $C_{1}=C_{2}=C$, then the system of generalized variational inequalities (SGVI) (27) reduces to SNVI (8) studied by Verma [14].

The system of generalized variational inequalities (27) is more general in nature. One can find various systems of generalized variational inequalities from SGVI (27).

We now discuss some special cases of (27) as follows.

Let $g_{i}: H \rightarrow H$ be single-valued $\delta_{i}$-strongly monotone, $\eta_{i}$-Lipschitz continuous, let $F_{i}: C_{i} \rightarrow H$ be $k_{i}$-Lipschitzian and $\xi_{i}$-strongly monotone operator with constants $k_{i}, \xi_{i}>0$, and let $V_{i}: C_{i} \rightarrow H$ be $L_{i}$-Lipschitzian mapping with constant $L_{i} \geq 0$ for $i \in\{1,2\}$. Suppose that $0<\mu_{i}<2 \xi_{i} / k_{i}^{2}$ and $0 \leq \gamma_{i}<\tau_{i} / L_{i}$, where $\tau_{i}=1-\sqrt{1-\mu_{i}\left(2 \xi_{i}-\mu_{i} k_{i}^{2}\right)}$ for $i \in\{1,2\}$.

If $T_{i}=\mu_{i} F_{i}-\gamma_{i} V_{i}$ for $i=1,2$, then the system of generalized variational inequalities (27) reduces to the following system of generalized variational inequalities (SGVI).

Find $\left(x^{*}, y^{*}\right) \in C_{1} \times C_{2}$ such that

$$
\begin{aligned}
& \left\langle s\left(\mu_{1} F_{1}-\gamma_{1} V_{1}\right)\left(x^{*}\right)+y^{*}-g_{1}\left(x^{*}\right), g_{1}(y)-y^{*}\right\rangle \\
& \quad \geq 0, \quad \forall y \in C_{2},
\end{aligned}
$$

$$
\begin{aligned}
& \left\langle t\left(\mu_{2} F_{2}-\gamma_{2} V_{2}\right)\left(y^{*}\right)+x^{*}-g_{2}\left(y^{*}\right), g_{2}(x)-x^{*}\right\rangle \\
& \quad \geq 0, \quad \forall x \in C_{1} .
\end{aligned}
$$

Define the mappings $S_{1}: C_{1} \rightarrow C_{2}$ and $S_{2}: C_{2} \rightarrow C_{1}$ by

$$
\begin{aligned}
& S_{1}:=P_{C_{2}}\left[g_{1}-s\left(\mu_{1} F_{1}-\gamma_{1} V_{1}\right)\right] \\
& S_{2}:=P_{C_{1}}\left[g_{2}-t\left(\mu_{2} F_{2}-\gamma_{2} V_{2}\right)\right],
\end{aligned}
$$

where $s$ and $t$ are some constants in $(0,1]$. Using Lemma 5, one can easily observe that the SGVI (28) is equivalent to the following altering point formulation:

to find $\left(x^{*}, y^{*}\right) \in C_{1}$

$$
\times C_{2} \text { such that }\left\{\begin{array}{l}
x^{*}=P_{C_{1}}\left[g_{2}-t\left(\mu_{2} F_{2}-\gamma_{2} V_{2}\right)\right]\left(y^{*}\right), \\
y^{*}=P_{C_{2}}\left[g_{1}-s\left(\mu_{1} F_{1}-\gamma_{1} V_{1}\right)\right]\left(x^{*}\right) .
\end{array}\right.
$$

First we introduce parallel Mann iteration process to solve system of generalized variational inequalities (28) as follows.

Algorithm 10. For any given $\left(x_{1}, y_{1}\right) \in C_{1} \times C_{2}$, let $\left\{\left(x_{n}, y_{n}\right)\right\}$ be an iterative sequence in $C_{1} \times C_{2}$ defined by

$$
\begin{aligned}
& x_{n+1}=\left(1-\alpha_{n}\right) x_{n}+\alpha_{n} S_{2}\left(y_{n}\right), \\
& y_{n+1}=\left(1-\alpha_{n}\right) y_{n}+\alpha_{n} S_{1}\left(x_{n}\right), \quad \forall n \in \mathbb{N},
\end{aligned}
$$

where $\left\{\alpha_{n}\right\}$ is a sequence in $[0,1]$ and $S_{1}$ and $S_{2}$ are defined by (29) and (30), respectively.

Motivated by Sahu [26] and equivalent formulation (31), we now propose a more general parallel $S$-iteration process to solve SGVI (28) as follows.

Algorithm 11. For any given $\left(x_{1}, y_{1}\right) \in C_{1} \times C_{2}$, let $\left\{\left(x_{n}, y_{n}\right)\right\}$ be an iterative sequence in $C_{1} \times C_{2}$ defined by

$$
\begin{aligned}
& x_{n+1}=S_{2}\left[\left(1-\alpha_{n}\right) y_{n}+\alpha_{n} S_{1}\left(x_{n}\right)\right], \\
& y_{n+1}=S_{1}\left[\left(1-\alpha_{n}\right) x_{n}+\alpha_{n} S_{2}\left(y_{n}\right)\right], \quad \forall n \in \mathbb{N},
\end{aligned}
$$

where $\left\{\alpha_{n}\right\}$ is a sequence in $(0,1)$ and $S_{1}$ and $S_{2}$ are defined by (29) and (30), respectively.

Before proving our main results, we will prove the following proposition which will be used in sequel.

Proposition 12. Let $C_{1}$ and $C_{2}$ be nonempty closed convex subsets of a real Hilbert space $H$. Let $g_{i}: H \rightarrow H$ be singlevalued $\delta_{i}$-strongly monotone, $\eta_{i}$-Lipschitz continuous, let $F_{i}$ : $C_{i} \rightarrow H$ be $k_{i}$-Lipschitzian and $\xi_{i}$-strongly monotone operator with constants $k_{i}, \xi_{i}>0$, and let $V_{i}: C_{i} \rightarrow H$ be $L_{i}$-Lipschitzian mapping with constant $L_{i} \geq 0$ for $i \in\{1,2\}$. Suppose that $0<\mu_{i}<2 \xi_{i} / k_{i}^{2}$ and $0 \leq \gamma_{i}<\tau_{i} / L_{i}$, where $\tau_{i}=$ $1-\sqrt{1-\mu_{i}\left(2 \xi_{i}-\mu_{i} k_{i}^{2}\right)}$ for $i \in\{1,2\}$. Let $s, t \in(0,1]$ and let $\theta_{1}$ and $\theta_{2}$ be real constants defined by

$$
\theta_{i}=\sqrt{1-2 \delta_{i}+\eta_{i}^{2}} \text { for } i=1,2 \text {. }
$$


Then the mappings $S_{1}$ and $S_{2}$ defined by (29) and (30) are Lipschitz continuous with Lipschitz constants $\left[\theta_{1}+\left(1-s\left(\tau_{1}-\right.\right.\right.$ $\left.\left.\left.\gamma_{1} L_{1}\right)\right)\right]$ and $\left[\theta_{2}+\left(1-t\left(\tau_{2}-\gamma_{2} L_{2}\right)\right)\right]$, respectively.

Proof. Let $x, y \in C_{1}$. Then, we have

$$
\begin{aligned}
& \left\|S_{1}(x)-S_{1}(y)\right\|=\| P_{C_{2}}\left[g_{1}-s\left(\mu_{1} F_{1}-\gamma_{1} V_{1}\right)\right](x) \\
& -P_{C_{2}}\left[g_{1}-s\left(\mu_{1} F_{1}-\gamma_{1} V_{1}\right)\right](y)\|\leq\| g_{1}(x) \\
& -s\left(\mu_{1} F_{1}-\gamma_{1} V_{1}\right)(x)-g_{1}(y) \\
& +s\left(\mu_{1} F_{1}-\gamma_{1} V_{1}\right)(y)\|=\| g_{1}(x)-g_{1}(y) \\
& -(x-y)+(x-y)-s\left(\mu_{1} F_{1}-\gamma_{1} V_{1}\right)(x-y) \| \\
& \leq\left\|g_{1}(x)-g_{1}(y)-(x-y)\right\|+\|(x-y) \\
& -s\left(\mu_{1} F_{1}-\gamma_{1} V_{1}\right)(x-y)\|\leq\| x-y \\
& -\left(g_{1}(x)-g_{1}(y)\right)\|+\| x-s \mu_{1} F_{1}(x)-y \\
& +s \mu_{1} F_{1}(y)\|+\| s \gamma_{1} V_{1}(x)-s \gamma_{1} V_{1}(y)\|\leq\| x-y \\
& -\left(g_{1}(x)-g_{1}(y)\right)\|+\|\left(I-s \mu_{1} F_{1}\right)(x) \\
& -\left(I-s \mu_{1} F_{1}\right)(y)\left\|+s \gamma_{1}\right\| V_{1}(x)-V_{1}(y)\|\leq\| x \\
& -y-\left(g_{1}(x)-g_{1}(y)\right)\left\|+\left(1-s \tau_{1}\right)\right\| x-y \| \\
& +s \gamma_{1} L_{1}\|x-y\|=\left\|x-y-\left(g_{1}(x)-g_{1}(y)\right)\right\| \\
& +\left(1-s\left(\tau_{1}-\gamma_{1} L_{1}\right)\right)\|x-y\|, \\
& \left\|x-y-\left(g_{1}(x)-g_{1}(y)\right)\right\|^{2}=\|x-y\|^{2}-2\langle x \\
& \left.-y, g_{1}(x)-g_{1}(y)\right\rangle+\left\|g_{1}(x)-g_{1}(y)\right\|^{2} \leq \| x \\
& -y\left\|^{2}-2 \delta_{1}\right\| x-y\left\|^{2}+\eta_{1}^{2}\right\| x-y \|^{2}=\left(1-2 \delta_{1}\right. \\
& \left.+\eta_{1}^{2}\right)\|x-y\|^{2}=\theta_{1}^{2}\|x-y\|^{2} \text {. }
\end{aligned}
$$

From (35), we have

$$
\begin{aligned}
& \left\|S_{1}(x)-S_{1}(y)\right\| \\
& \quad \leq \theta_{1}\|x-y\|+\left(1-s\left(\tau_{1}-\gamma_{1} L_{1}\right)\right)\|x-y\| \\
& \quad=\left[\theta_{1}+\left(1-s\left(\tau_{1}-\gamma_{1} L_{1}\right)\right)\right]\|x-y\| .
\end{aligned}
$$

Thus $S_{1}$ is $\left[\theta_{1}+\left(1-s\left(\tau_{1}-\gamma_{1} L_{1}\right)\right)\right]$-Lipschitz continuous.

Similarly, we can show that $S_{2}$ is $\left[\theta_{2}+\left(1-t\left(\tau_{2}-\gamma_{2} L_{2}\right)\right)\right]-$ Lipschitz continuous.

Now we are ready to present our main results. First we establish the convergence analysis of Algorithm 10 for solving SGVI (28).

Theorem 13. Let $C_{1}$ and $C_{2}$ be nonempty closed convex subsets of a real Hilbert space $H$. Let $g_{i}: H \rightarrow H$ be single-valued $\delta_{i}$-strongly monotone, $\eta_{i}$-Lipschitz continuous, let $F_{i}: C_{i} \rightarrow$ $H$ be $k_{i}$-Lipschitzian and $\xi_{i}$-strongly monotone operator with constants $k_{i}, \xi_{i}>0$, and let $V_{i}: C_{i} \rightarrow H$ be $L_{i}$-Lipschitzian mapping with constant $L_{i} \geq 0$ for $i \in\{1,2\}$. Suppose that $0<\mu_{i}<2 \xi_{i} / k_{i}^{2}$ and $0 \leq \gamma_{i}<\tau_{i} / L_{i}$, where $\tau_{i}=1-$ $\sqrt{1-\mu_{i}\left(2 \xi_{i}-\mu_{i} k_{i}^{2}\right)}$ for $i \in\{1,2\}$. Let $s, t \in(0,1]$ and let $\theta_{1}$ and $\theta_{2}$ be real constants defined by (34). Let $S_{1}$ and $S_{2}$ be defined by (29) and (30), respectively. For given initial point $\left(x_{1}, y_{1}\right) \in C_{1} \times C_{2}$, let $\left\{\left(x_{n}, y_{n}\right)\right\}$ be an iterative sequence defined by parallel Mann iteration process (32), where $\left\{\alpha_{n}\right\}$ is a sequence in $[0,1]$ such that $\sum_{n=1}^{\infty} \alpha_{n}=\infty$. Assume that the following condition is satisfied:

$$
\begin{aligned}
& \frac{\theta_{1}}{s}<\left(\tau_{1}-\gamma_{1} L_{1}\right), \\
& \frac{\theta_{2}}{t}<\left(\tau_{2}-\gamma_{2} L_{2}\right) .
\end{aligned}
$$

Then we have the following:

(i) There exists a unique point $\left(x^{*}, y^{*}\right) \in C_{1} \times C_{2}$, which solves SGVI (28).

(ii) The sequence $\left\{\left(x_{n}, y_{n}\right)\right\}$ generated by parallel Mann iteration process (32) converges strongly to the point $\left(x^{*}, y^{*}\right)$.

Proof. (i) It follows from Lemma 8 and (31).

(ii) By (31), (32), and Proposition 12, we have

$$
\begin{aligned}
&\left\|x_{n+1}-x^{*}\right\| \\
&=\left\|\left(1-\alpha_{n}\right) x_{n}+\alpha_{n} S_{2}\left(y_{n}\right)-\left(1-\alpha_{n}\right) x^{*}-\alpha_{n} x^{*}\right\| \\
& \leq\left(1-\alpha_{n}\right)\left\|x_{n}-x^{*}\right\|+\alpha_{n}\left\|S_{2}\left(y_{n}\right)-x^{*}\right\| \\
&=\left(1-\alpha_{n}\right)\left\|x_{n}-x^{*}\right\|+\alpha_{n}\left\|S_{2}\left(y_{n}\right)-S_{2}\left(y^{*}\right)\right\| \\
& \leq\left(1-\alpha_{n}\right)\left\|x_{n}-x^{*}\right\| \\
&+\alpha_{n}\left[\theta_{2}+\left(1-t\left(\tau_{2}-\gamma_{2} L_{2}\right)\right)\right]\left\|y_{n}-y^{*}\right\| .
\end{aligned}
$$

Again, by Proposition 12 that $S_{1}: C_{1} \rightarrow C_{2}$ is $\left[\theta_{1}+\left(1-s\left(\tau_{1}-\right.\right.\right.$ $\left.\gamma_{1} L_{1}\right)$ )]-Lipschitz continuous and using (31) and (32), we have

$$
\begin{aligned}
& \left\|y_{n+1}-y^{*}\right\| \\
& \quad \leq\left(1-\alpha_{n}\right)\left\|y_{n}-y^{*}\right\| \\
& \quad+\alpha_{n}\left[\theta_{1}+\left(1-s\left(\tau_{1}-\gamma_{1} L_{1}\right)\right)\right]\left\|x_{n}-x^{*}\right\| .
\end{aligned}
$$

Set

$$
\begin{aligned}
\theta= & \max \left\{\theta_{1}+\left(1-s\left(\tau_{1}-\gamma_{1} L_{1}\right)\right), \theta_{2}\right. \\
& \left.+\left(1-t\left(\tau_{2}-\gamma_{2} L_{2}\right)\right)\right\} .
\end{aligned}
$$

From (38) and (39), we get

$$
\begin{aligned}
& \left\|x_{n+1}-x^{*}\right\|+\left\|y_{n+1}-y^{*}\right\| \\
& \quad \leq\left(1-\alpha_{n}\right)\left\|x_{n}-x^{*}\right\| \\
& \quad+\alpha_{n}\left[\theta_{2}+\left(1-t\left(\tau_{2}-\gamma_{2} L_{2}\right)\right)\right]\left\|y_{n}-y^{*}\right\|
\end{aligned}
$$




$$
\begin{aligned}
& +\left(1-\alpha_{n}\right)\left\|y_{n}-y^{*}\right\| \\
& +\alpha_{n}\left[\theta_{1}+\left(1-s\left(\tau_{1}-\gamma_{1} L_{1}\right)\right)\right]\left\|x_{n}-x^{*}\right\| \\
\leq & \left(1-\alpha_{n}\right)\left\|x_{n}-x^{*}\right\|+\alpha_{n} \theta\left\|y_{n}-y^{*}\right\| \\
& +\left(1-\alpha_{n}\right)\left\|y_{n}-y^{*}\right\|+\alpha_{n} \theta\left\|x_{n}-x^{*}\right\| \\
= & \left(1-\alpha_{n}(1-\theta)\right)\left(\left\|x_{n}-x^{*}\right\|+\left\|y_{n}-y^{*}\right\|\right) .
\end{aligned}
$$

Now, we define the norm $\|\cdot\|_{1}$ on $H \times H$ by $\|(x, y)\|_{1}=$ $\|x\|+\|y\|$ for all $(x, y) \in H \times H$. Therefore, using (41), we have

$$
\begin{aligned}
& \left\|\left(x_{n+1}, y_{n+1}\right)-\left(x^{*}, y^{*}\right)\right\|_{1} \\
& \quad=\left\|\left(x_{n+1}-x^{*}, y_{n+1}-y^{*}\right)\right\|_{1} \\
& \quad=\left\|x_{n+1}-x^{*}\right\|+\left\|y_{n+1}-y^{*}\right\| \\
& \quad \leq\left(1-\alpha_{n}(1-\theta)\right)\left(\left\|x_{n}-x^{*}\right\|+\left\|y_{n}-y^{*}\right\|\right) \\
& \quad=\left(1-\alpha_{n}(1-\theta)\right)\left\|\left(x_{n}, y_{n}\right)-\left(x^{*}, y^{*}\right)\right\|_{1} .
\end{aligned}
$$

Noticing that $\sum_{n=1}^{\infty} \alpha_{n}=\infty$ and $\theta \in(0,1)$. Therefore, from Lemma 7, we have $\lim _{n \rightarrow \infty}\left\|\left(x_{n}, y_{n}\right)-\left(x^{*}, y^{*}\right)\right\|_{1}=0$. Thus, we get $\lim _{n \rightarrow \infty}\left\|x_{n}-x^{*}\right\|=\lim _{n \rightarrow \infty}\left\|y_{n}-y^{*}\right\|=0$ and hence $\left\{x_{n}\right\}$ and $\left\{y_{n}\right\}$ converge to $x^{*}$ and $y^{*}$, respectively.

Corollary 14. Let $C_{1}$ and $C_{2}$ be nonempty closed convex subsets of a real Hilbert space $H$. Let $F_{i}: C_{i} \rightarrow H$ be $k_{i}-$ Lipschitzian and $\xi_{i}$-strongly monotone operator with constants $k_{i}, \xi_{i}>0$ and let $V_{i}: C_{i} \rightarrow H$ be $L_{i}$-Lipschitzian mapping with constant $L_{i} \geq 0$ for $i \in\{1,2\}$. Suppose that $0<\mu_{i}<2 \xi_{i} / k_{i}^{2}$ and $0 \leq \gamma_{i}<\tau_{i} / L_{i}$, where $\tau_{i}=1-\sqrt{1-\mu_{i}\left(2 \xi_{i}-\mu_{i} k_{i}^{2}\right)}$ for $i \in\{1,2\}$. Let $s, t \in(0,1]$. Define mappings $S_{1}: C_{1} \rightarrow C_{2}$ and $S_{2}: C_{2} \rightarrow C_{1}$ by

$$
\begin{aligned}
& S_{1}:=P_{C_{2}}\left[I-s\left(\mu_{1} F_{1}-\gamma_{1} V_{1}\right)\right], \\
& S_{2}:=P_{C_{1}}\left[I-t\left(\mu_{2} F_{2}-\gamma_{2} V_{2}\right)\right] .
\end{aligned}
$$

For given initial point $\left(x_{1}, y_{1}\right) \in C_{1} \times C_{2}$, let $\left\{\left(x_{n}, y_{n}\right)\right\}$ be an iterative sequence in $C_{1} \times C_{2}$ defined by

$$
\begin{aligned}
& x_{n+1}=\left(1-\alpha_{n}\right) x_{n}+\alpha_{n} S_{2}\left(y_{n}\right), \\
& y_{n+1}=\left(1-\alpha_{n}\right) y_{n}+\alpha_{n} S_{1}\left(x_{n}\right), \quad \forall n \in \mathbb{N},
\end{aligned}
$$

where $\left\{\alpha_{n}\right\}$ is a real sequence in $[0,1]$ such that $\sum_{n=1}^{\infty} \alpha_{n}=\infty$. Assume that condition (37) of Theorem 13 is satisfied. Then the sequence $\left\{\left(x_{n}, y_{n}\right)\right\}$ generated by (44) converges strongly to the unique point $\left(x^{*}, y^{*}\right)$, which solves system of generalized variational inequalities

$$
\begin{aligned}
& \left\langle s\left(\mu_{1} F_{1}-\gamma_{1} V_{1}\right)\left(x^{*}\right)+y^{*}-x^{*}, y-y^{*}\right\rangle \geq 0, \\
& \forall y \in C_{2} \text {, } \\
& \left\langle t\left(\mu_{2} F_{2}-\gamma_{2} V_{2}\right)\left(y^{*}\right)+x^{*}-y^{*}, x-x^{*}\right\rangle \geq 0,
\end{aligned}
$$

Proof. The proof follows from Theorem 13 by taking $g_{1}=$ $g_{2}=I$.

Now we study the convergence analysis of Algorithm 11, that is, the parallel $S$-iteration process defined by (33) for solving SGVI (28).

Theorem 15. Let $C_{1}$ and $C_{2}$ be nonempty closed convex subsets of a real Hilbert space $H$. Let $g_{i}: H \rightarrow H$ be single-valued $\delta_{i}$-strongly monotone, $\eta_{i}$-Lipschitz continuous, let $F_{i}: C_{i} \rightarrow$ $H$ be $k_{i}$-Lipschitzian and $\xi_{i}$-strongly monotone operator with constants $k_{i}, \xi_{i}>0$, and let $V_{i}: C_{i} \rightarrow H$ be $L_{i}$-Lipschitzian mapping with constant $L_{i} \geq 0$ for $i \in\{1,2\}$. Suppose that $0<\mu_{i}<2 \xi_{i} / k_{i}^{2}$ and $0 \leq \gamma_{i}<\tau_{i} / L_{i}$, where $\tau_{i}=1-$ $\sqrt{1-\mu_{i}\left(2 \xi_{i}-\mu_{i} k_{i}^{2}\right)}$ for $i \in\{1,2\}$. Let $s, t \in(0,1]$ and let $\theta_{1}$ and $\theta_{2}$ be real constants defined by (34). Let $S_{1}$ and $S_{2}$ be defined by (29) and (30), respectively. For given initial point $\left(x_{1}, y_{1}\right) \in C_{1} \times C_{2}$, let $\left\{\left(x_{n}, y_{n}\right)\right\}$ be an iterative sequence in $C_{1} \times C_{2}$ defined by parallel $S$-iteration process (33), where $\left\{\alpha_{n}\right\}$ is a sequence in $(0,1)$. Assume that condition (37) of Theorem 13 is satisfied. Then we have the following:

(i) There exists a unique point $\left(x^{*}, y^{*}\right) \in C_{1} \times C_{2}$, which solves SGVI (28).

(ii) The sequence $\left\{\left(x_{n}, y_{n}\right)\right\}$ generated by parallel $S$ iteration process (33) converges strongly to the point $\left(x^{*}, y^{*}\right)$.

Proof. (i) It follows from Lemma 8 and (31).

(ii) From (31), (33), and Proposition 12, we have

$$
\begin{aligned}
& \left\|y_{n+1}-y^{*}\right\|=\| S_{1}\left[\left(1-\alpha_{n}\right) x_{n}+\alpha_{n} S_{2}\left(y_{n}\right)\right] \\
& -S_{1}\left(x^{*}\right) \| \leq\left(\theta_{1}+\left(1-s\left(\tau_{1}-\gamma_{1} L_{1}\right)\right)\right) \\
& \cdot\left\|\left(1-\alpha_{n}\right) x_{n}+\alpha_{n} S_{2}\left(y_{n}\right)-x^{*}\right\| \leq\left(\theta_{1}\right. \\
& \left.+\left(1-s\left(\tau_{1}-\gamma_{1} L_{1}\right)\right)\right)\left\{\left(1-\alpha_{n}\right)\left\|x_{n}-x^{*}\right\|\right. \\
& \left.+\alpha_{n}\left\|S_{2}\left(y_{n}\right)-x^{*}\right\|\right\}=\left(\theta_{1}+\left(1-s\left(\tau_{1}-\gamma_{1} L_{1}\right)\right)\right) \\
& \cdot\left\{\left(1-\alpha_{n}\right)\left\|x_{n}-x^{*}\right\|+\alpha_{n}\left\|S_{2}\left(y_{n}\right)-S_{2}\left(y^{*}\right)\right\|\right\} \\
& \quad \leq\left(\theta_{1}+\left(1-s\left(\tau_{1}-\gamma_{1} L_{1}\right)\right)\right)\left\{\left(1-\alpha_{n}\right)\left\|x_{n}-x^{*}\right\|\right. \\
& \left.+\alpha_{n}\left(\theta_{2}+\left(1-t\left(\tau_{2}-\gamma_{2} L_{2}\right)\right)\right)\left\|y_{n}-y^{*}\right\|\right\} .
\end{aligned}
$$

Similarly

$$
\begin{aligned}
& \left\|x_{n+1}-x^{*}\right\| \leq\left(\theta_{2}+\left(1-t\left(\tau_{2}-\gamma_{2} L_{2}\right)\right)\right) \\
& \cdot\left\{\left(1-\alpha_{n}\right)\left\|y_{n}-y^{*}\right\|\right. \\
& \left.\quad+\alpha_{n}\left(\theta_{1}+\left(1-s\left(\tau_{1}-\gamma_{1} L_{1}\right)\right)\right)\left\|x_{n}-x^{*}\right\|\right\} .
\end{aligned}
$$

Set

$$
\begin{aligned}
\theta= & \max \left\{\theta_{1}+\left(1-s\left(\tau_{1}-\gamma_{1} L_{1}\right)\right), \theta_{2}\right. \\
& \left.+\left(1-t\left(\tau_{2}-\gamma_{2} L_{2}\right)\right)\right\} .
\end{aligned}
$$


From (46) and (47), we get

$$
\begin{aligned}
& \left\|x_{n+1}-x^{*}\right\|+\left\|y_{n+1}-y^{*}\right\| \leq\left(\theta_{2}\right. \\
& \left.\quad+\left(1-t\left(\tau_{2}-\gamma_{2} L_{2}\right)\right)\right)\left\{\left(1-\alpha_{n}\right)\left\|y_{n}-y^{*}\right\|\right. \\
& \left.\quad+\alpha_{n}\left(\theta_{1}+\left(1-s\left(\tau_{1}-\gamma_{1} L_{1}\right)\right)\right)\left\|x_{n}-x^{*}\right\|\right\}+\left(\theta_{1}\right. \\
& \left.\quad+\left(1-s\left(\tau_{1}-\gamma_{1} L_{1}\right)\right)\right)\left\{\left(1-\alpha_{n}\right)\left\|x_{n}-x^{*}\right\|\right. \\
& \left.\quad+\alpha_{n}\left(\theta_{2}+\left(1-t\left(\tau_{2}-\gamma_{2} L_{2}\right)\right)\right)\left\|y_{n}-y^{*}\right\|\right\} \\
& \quad \leq \theta\left\{\left(1-\alpha_{n}\right)\left\|y_{n}-y^{*}\right\|+\alpha_{n} \theta\left\|x_{n}-x^{*}\right\|\right\} \\
& \quad+\theta\left\{\left(1-\alpha_{n}\right)\left\|x_{n}-x^{*}\right\|+\alpha_{n} \theta\left\|y_{n}-y^{*}\right\|\right\}=\theta(1 \\
& \left.-\alpha_{n}(1-\theta)\right)\left\|x_{n}-x^{*}\right\|+\theta\left(1-\alpha_{n}(1-\theta)\right) \| y_{n} \\
& -y^{*} \|=\theta\left(1-\alpha_{n}(1-\theta)\right)\left[\left\|x_{n}-x^{*}\right\|+\left\|y_{n}-y^{*}\right\|\right] .
\end{aligned}
$$

Now, we define the norm $\|\cdot\|_{1}$ on $H \times H$ by $\|(x, y)\|_{1}=\|x\|+$ $\|y\|$ for all $(x, y) \in H \times H$. Therefore, using (49), we have

$$
\begin{aligned}
& \left\|\left(x_{n+1}, y_{n+1}\right)-\left(x^{*}, y^{*}\right)\right\|_{1} \\
& \quad=\left\|\left(x_{n+1}-x^{*}, y_{n+1}-y^{*}\right)\right\|_{1} \\
& \quad=\left\|x_{n+1}-x^{*}\right\|+\left\|y_{n+1}-y^{*}\right\| \\
& \quad \leq \theta\left(1-\alpha_{n}(1-\theta)\right)\left[\left\|x_{n}-x^{*}\right\|+\left\|y_{n}-y^{*}\right\|\right] \\
& \quad=\theta\left(1-\alpha_{n}(1-\theta)\right)\left\|\left(x_{n}, y_{n}\right)-\left(x^{*}, y^{*}\right)\right\|_{1} .
\end{aligned}
$$

Since $\theta\left(1-\alpha_{n}(1-\theta)\right) \leq \theta<1$, we obtain that $\lim _{n \rightarrow \infty}\left\|\left(x_{n}, y_{n}\right)-\left(x^{*}, y^{*}\right)\right\|_{1}=0$. Thus, we get $\lim _{n \rightarrow \infty} \| x_{n}-$ $x^{*}\left\|=\lim _{n \rightarrow \infty}\right\| y_{n}-y^{*} \|=0$ and hence $\left\{x_{n}\right\}$ and $\left\{y_{n}\right\}$ converge to $x^{*}$ and $y^{*}$, respectively.

Corollary 16. Let $C_{1}$ and $C_{2}$ be nonempty closed convex subsets of a real Hilbert space $H$. Let $F_{i}: C_{i} \rightarrow H$ be $k_{i}-$ Lipschitzian and $\xi_{i}$-strongly monotone operator with constants $k_{i}, \xi_{i}>0$ and let $V_{i}: C_{i} \rightarrow H$ be $L_{i}$-Lipschitzian mapping with constant $L_{i} \geq 0$ for $i \in\{1,2\}$. Suppose that $0<\mu_{i}<2 \xi_{i} / k_{i}^{2}$ and $0 \leq \gamma_{i}<\tau_{i} / L_{i}$, where $\tau_{i}=1-\sqrt{1-\mu_{i}\left(2 \xi_{i}-\mu_{i} k_{i}^{2}\right)}$ for $i \in\{1,2\}$. Let $s, t \in(0,1]$ and let $S_{1}$ and $S_{2}$ be defined by (43). For given initial point $\left(x_{1}, y_{1}\right) \in C_{1} \times C_{2}$, let $\left\{\left(x_{n}, y_{n}\right)\right\}$ be an iterative sequence in $C_{1} \times C_{2}$ defined by

$$
\begin{aligned}
& x_{n+1}=S_{2}\left[\left(1-\alpha_{n}\right) y_{n}+\alpha_{n} S_{1}\left(x_{n}\right)\right], \\
& y_{n+1}=S_{1}\left[\left(1-\alpha_{n}\right) x_{n}+\alpha_{n} S_{2}\left(y_{n}\right)\right], \quad \forall n \in \mathbb{N},
\end{aligned}
$$

where $\left\{\alpha_{n}\right\}$ is a real sequence in $(0,1)$. Assume that condition (37) of Theorem 13 is satisfied. Then the sequence $\left\{\left(x_{n}, y_{n}\right)\right\}$ generated by (51) converges strongly to the unique point $\left(x^{*}, y^{*}\right)$, which solves system of generalized variational inequalities

$$
\begin{aligned}
& \left\langle s\left(\mu_{1} F_{1}-\gamma_{1} V_{1}\right)\left(x^{*}\right)+y^{*}-x^{*}, y-y^{*}\right\rangle \geq 0, \\
& \forall y \in C_{2} \text {, } \\
& \left\langle t\left(\mu_{2} F_{2}-\gamma_{2} V_{2}\right)\left(y^{*}\right)+x^{*}-y^{*}, x-x^{*}\right\rangle \geq 0,
\end{aligned}
$$

Proof. The proof follows from Theorem 15 by taking $g_{1}=$ $g_{2}=I$.

\section{Numerical Example}

In this section, we discuss an example which leads to Theorems 13 and 15. The graphs are also presented for showing how the sequences $\left\{x_{n}\right\}$ and $\left\{y_{n}\right\}$ generated by both the algorithms, Algorithms 10 and 11, converge to the solutions of SGVI (28).

Example 17. Let $H=\mathbb{R}, C_{1}=(-\infty, 0]$, and $C_{2}=[0, \infty)$. Let $g_{1}$ and $g_{2}$ be two mappings from $H$ onto itself defined by $g_{1}(x)=(2 x-3) / 3$ for all $x \in H$ and $g_{2}(x)=(5 x-10) / 6$ for all $x \in H$, respectively. Let $F_{1}: C_{1} \rightarrow H$ and $F_{2}: C_{2} \rightarrow H$ be two mappings defined by $F_{1}(x)=2 x-3$ for all $x \in C_{1}$ and $F_{2}(x)=3 x-2$ for all $x \in C_{2}$, respectively. Let $V_{1}: C_{1} \rightarrow H$ and $V_{2}: C_{2} \rightarrow H$ be two mappings defined by $V_{1}(x)=1-4 x$ for all $x \in C_{1}$ and $V_{2}(x)=6-6 x$ for all $x \in C_{2}$, respectively. Then $g_{i}$ is $\delta_{i}$-strongly monotone and $\eta_{i}$-Lipschitzian mapping for $i \in\{1,2\}$. We have $\delta_{1}=2 / 3=\eta_{1}$ and $\delta_{2}=5 / 6=\eta_{2}$. Also $F_{i}$ is $\xi_{i}$-strongly monotone and $k_{i}$-Lipschitzian mapping for $i \in\{1,2\}$. We have $\xi_{1}=2=k_{1}$ and $\xi_{2}=3=k_{2}$. Moreover $V_{i}$ is $L_{i}$-Lipschitzian mapping for $i \in\{1,2\}$. We have $L_{1}=4$ and $L_{2}=6$. We take $\mu_{1}=1 / 2, \mu_{2}=1 / 3, \tau_{1}=1=\tau_{2}$ and $\gamma_{1}=1 / 12, \gamma_{2}=5 / 72$. Define $\left\{\alpha_{n}\right\}$ in $[0,1]$ by $\alpha_{n}=n /(n+1)$, $T_{1}=\mu_{1} F_{1}-\gamma_{1} V_{1}$, and $T_{2}=\mu_{2} F_{2}-\gamma_{2} V_{2}$. Then $T_{1}(x)=(16 x-$ 19) $/ 12$ and $T_{2}(x)=(51 x-39) / 36$.

Therefore $S_{1}$ and $S_{2}$ can be expressed as

$$
\begin{aligned}
& S_{1}:=P_{C_{2}}\left[g_{1}-s T_{1}\right], \\
& S_{2}:=P_{C_{1}}\left[g_{2}-t T_{2}\right],
\end{aligned}
$$

where $s>0$ and $t>0$ are constants.

$$
\begin{aligned}
& \text { Let } s=1 \text { and } t=1 \text {. Then, } \\
& S_{1}(x)=P_{C_{2}}\left[\frac{(-8 x+7)}{12}\right]=\frac{(7-8 x)}{12} \quad \forall x \in C_{1}, \\
& S_{2}(x)=P_{C_{1}}\left[\frac{(-7 x-7)}{12}\right]=-\frac{(7 x+7)}{12} \quad \forall x \in C_{2} .
\end{aligned}
$$

It can be easily seen that $S_{1}: C_{1} \rightarrow C_{2}$ is (2/3)-Lipschitzian and $S_{2}: C_{2} \rightarrow C_{1}$ is (7/12)-Lipschitzian. Also

$$
\begin{aligned}
\frac{\theta_{1}}{s}-\left(\tau_{1}-\gamma_{1} L_{1}\right) & =\sqrt{1-2 \delta_{1}+\eta_{1}^{2}}-\left(\tau_{1}-\gamma_{1} L_{1}\right) \\
& =-\frac{1}{3}<0, \\
\frac{\theta_{2}}{t}-\left(\tau_{2}-\gamma_{2} L_{2}\right) & =\sqrt{1-2 \delta_{2}+\eta_{2}^{2}}-\left(\tau_{2}-\gamma_{2} L_{2}\right) \\
& =-\frac{5}{12}<0 .
\end{aligned}
$$

One can observe that all the conditions of Theorems 13 and 15 are satisfied. 
TABLE 1: Numerical values of $x_{n}$ and $y_{n}$.

\begin{tabular}{lcccc}
\hline$n$ & \multicolumn{2}{c}{ Parallel Mann iteration process } & \multicolumn{2}{c}{ Parallel S-iteration process } \\
& $x_{n}$ & $y_{n}$ & $x_{n}$ & $y_{n}$ \\
\hline 1 & -4.000000000000000 & 4.000000000000000 & -4.000000000000000 & 4.000000000000000 \\
7 & -1.847585060258671 & 1.950379984812855 & -1.531137975937710 & 1.612050811863993 \\
14 & -1.529511272915085 & 1.610309552955496 & -1.511402781884560 & 1.590950939614087 \\
21 & -1.512204443955688 & 1.591807954179574 & -1.511363702883595 & 1.590909162022088 \\
28 & -1.511400117495092 & 1.590948090842850 & -1.511363636469492 & 1.590909091022255 \\
35 & -1.511365162111111 & 1.590910722002483 & -1.511363636363799 & 1.590909090909265 \\
42 & -1.511363698691403 & 1.590909157540259 & -1.511363636363637 & 1.590909090909091 \\
49 & -1.511363638868444 & 1.590909093586843 & -1.511363636363637 & 1.590909090909091 \\
56 & -1.511363636463094 & 1.590909091015415 & -1.511363636363636 & 1.590909090909091 \\
63 & -1.511363636367549 & 1.590909090913274 & -1.511363636363637 & 1.590909090909091 \\
70 & -1.511363636363790 & 1.590909090909254 & -1.511363636363637 & 1.590909090909091 \\
77 & -1.511363636363642 & 1.590909090909097 & -1.511363636363637 & 1.590909090909091 \\
84 & -1.511363636363637 & 1.590909090909091 & -1.511363636363636 & 1.590909090909091 \\
91 & -1.511363636363637 & 1.590909090909091 & -1.511363636363637 & 1.590909090909091 \\
98 & -1.511363636363637 & 1.590909090909091 & -1.511363636363637 & 1.590909090909091 \\
\hline
\end{tabular}

Now we will find the general term of the sequences $\left\{x_{n}\right\}$ and $\left\{y_{n}\right\}$ generated by the iteration process (32). For arbitrary $x_{1} \in C_{1}$ and $y_{1} \in C_{2}$,

$$
\begin{aligned}
x_{n+1} & =\left(1-\alpha_{n}\right) x_{n}+\alpha_{n} S_{2}\left(y_{n}\right) \\
& =\frac{x_{n}}{n+1}-\frac{n\left(7 y_{n}+7\right)}{12(n+1)} \\
& =\frac{1}{12(n+1)}\left[12 x_{n}-7 n y_{n}-7 n\right], \\
y_{n+1} & =\left(1-\alpha_{n}\right) y_{n}+\alpha_{n} S_{1}\left(x_{n}\right) \\
& =\frac{y_{n}}{n+1}+\frac{n\left(7-8 x_{n}\right)}{12(n+1)} \\
& =\frac{1}{12(n+1)}\left[12 y_{n}-8 n x_{n}+7 n\right] .
\end{aligned}
$$

Hence

$$
\begin{aligned}
& x_{n+1}=\frac{1}{12(n+1)}\left[12 x_{n}-7 n y_{n}-7 n\right], \\
& y_{n+1}=\frac{1}{12(n+1)}\left[12 y_{n}-8 n x_{n}+7 n\right] \quad \forall n \in \mathbb{N} .
\end{aligned}
$$

Also, we will find the general term of the sequences $\left\{x_{n}\right\}$ and $\left\{y_{n}\right\}$ generated by the iteration process (33). For arbitrary $x_{1} \in$ $C_{1}$ and $y_{1} \in C_{2}$,

$$
\begin{aligned}
x_{n+1} & =S_{2}\left[\left(1-\alpha_{n}\right) y_{n}+\alpha_{n} S_{1}\left(x_{n}\right)\right] \\
& =S_{2}\left[\frac{y_{n}}{n+1}+\frac{n\left(7-8 x_{n}\right)}{12(n+1)}\right] \\
& =\frac{1}{144(n+1)}\left[56 n x_{n}-84 y_{n}-133 n-84\right],
\end{aligned}
$$

$$
\begin{aligned}
y_{n+1} & =S_{1}\left[\left(1-\alpha_{n}\right) x_{n}+\alpha_{n} S_{2}\left(y_{n}\right)\right] \\
& =S_{1}\left[\frac{x_{n}}{n+1}-\frac{n\left(7 y_{n}+7\right)}{12(n+1)}\right] \\
& =\frac{1}{144(n+1)}\left[56 n y_{n}-96 x_{n}+140 n+84\right] .
\end{aligned}
$$

Hence

$$
\begin{aligned}
& x_{n+1}=\frac{1}{144(n+1)}\left[56 n x_{n}-84 y_{n}-133 n-84\right], \\
& y_{n+1}=\frac{1}{144(n+1)}\left[56 n y_{n}-96 x_{n}+140 n+84\right]
\end{aligned}
$$

$\forall n \in \mathbb{N}$.

It is clear from (57) and (59) that the sequences $\left\{x_{n}\right\}$ and $\left\{y_{n}\right\}$ generated by the proposed iterative algorithms converge to the altering points $x^{*} \in C_{1}$ and $y^{*} \in C_{2}$ of the mappings $S_{1}$ and $S_{2}$, where $x^{*}=-1.511363636363637$ and $y^{*}=$ 1.590909090909091. The numerical values of $\left\{x_{n}\right\}$ and $\left\{y_{n}\right\}$ have been calculated for different starting values of $x_{1}$ and $y_{1}$ in Tables 1 and 2, respectively, and the convergence of both the sequences is shown in Figures 1 and 2, respectively.

\section{Conclusions}

In this paper, we have considered a new system of generalized variational inequalities (SGVI) defined on closed convex subsets of a real Hilbert space. It has been shown that the considered SGVI is equivalent to altering points problem of some nonlinear mappings. We have proposed two algorithms, Algorithms 10 and 11, for solving considered SGVI. An example is given in support of our main results. We observed that the sequence generated by Algorithm 11 
TABLE 2: Numerical values of $x_{n}$ and $y_{n}$

\begin{tabular}{lcccc}
\hline$n$ & \multicolumn{2}{c}{ Parallel Mann iteration process } & \multicolumn{2}{c}{ Parallel S-iteration process } \\
\hline 1 & $x_{n}$ & $y_{n}$ & $x_{n}$ & $y_{n}$ \\
7 & -5.000000000000000 & 10.000000000000000 & -5.000000000000000 & 10.000000000000000 \\
14 & -2.316760667744464 & 2.451261819028022 & -1.558731808819676 & 1.641509397106758 \\
21 & -1.554814798532934 & 1.637363641212413 & -1.511457370603911 & 1.591009289899863 \\
28 & -1.513376896656526 & 1.593061316754131 & -1.511363795641447 & 1.590909261181073 \\
35 & -1.511450986841399 & 1.591002473172358 & -1.511363636617098 & 1.590909091180052 \\
42 & -1.511367289637843 & 1.590912996409872 & -1.511363636364025 & 1.590909090909507 \\
49 & -1.511363785601782 & 1.590909250451687 & -1.511363636363637 & 1.590909090909092 \\
56 & -1.511363642361179 & 1.590909097320727 & -1.511363636363637 & 1.590909090909091 \\
63 & -1.511363636601778 & 1.590909091163675 & -1.511363636363636 & 1.590909090909091 \\
70 & -1.511363636373005 & 1.590909090919107 & -1.511363636363637 & 1.590909090909091 \\
77 & -1.511363636364002 & 1.590909090909482 & -1.511363636363637 & 1.590909090909091 \\
84 & -1.511363636363651 & 1.590909090909106 & -1.511363636363637 & 1.590909090909091 \\
91 & -1.511363636363637 & 1.590909090909092 & -1.511363636363636 & 1.590909090909091 \\
98 & -1.511363636363637 & 1.590909090909091 & -1.511363636363637 & 1.590909090909091 \\
\hline
\end{tabular}

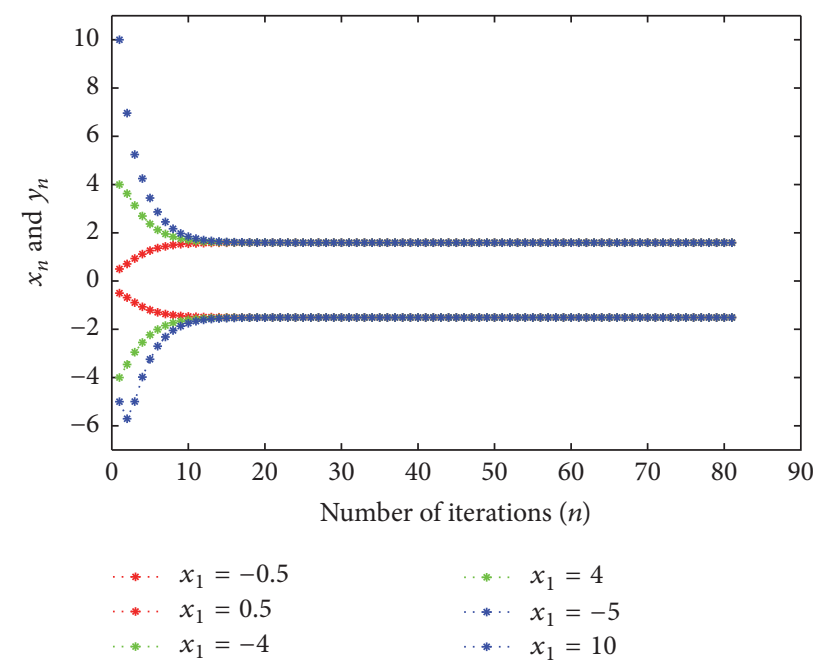

Figure 1: Convergence of sequences $\left\{x_{n}\right\}$ and $\left\{y_{n}\right\}$ generated by parallel Mann iteration process (32).

converges faster than Algorithm 10 to altering points of some nonlinear mappings $S_{1}$ and $S_{2}$.

\section{Conflicts of Interest}

The authors declare that there are no conflicts of interest regarding the publication of this paper.

\section{Acknowledgments}

The third author is supported by the Council of Scientific and Industrial Research (CSIR), New Delhi, India, through Grant 09/013(0584)/2015-EMR-I.

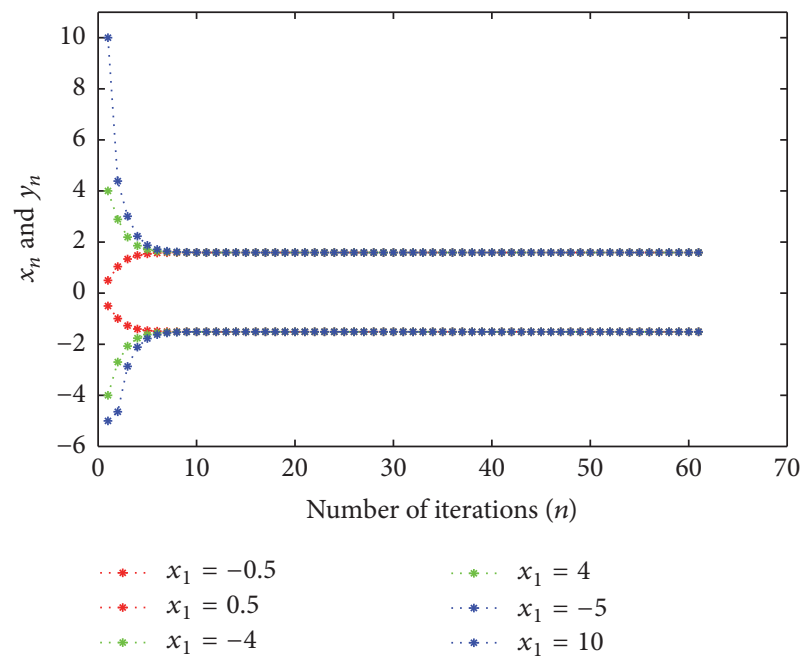

Figure 2: Convergence of sequences $\left\{x_{n}\right\}$ and $\left\{y_{n}\right\}$ generated by parallel $S$-iteration process (33).

\section{References}

[1] G. Stampacchia, "Formes bilineaires coercivites sur les ensembles convexes," Comptes Rendus de l'Académie des Sciences, vol. 258, pp. 4413-4416, 1964.

[2] E. Zeidler, Nonlinear Functional Analysis and Its Applications, Springer, New York, NY, USA, 1985.

[3] R. P. Agarwal, D. O’Regan, and D. R. Sahu, "Iterative construction of fixed points of nearly asymptotically nonexpansive mappings," Journal of Nonlinear and Convex Analysis, vol. 8, no. 1, pp. 61-79, 2007.

[4] D. R. Sahu, "Applications of the $S$-iteration process to constrained minimization problems and split feasibility problems," Fixed Point Theory, vol. 12, no. 1, pp. 187-204, 2011. 
[5] E. Picard, "Mémoire sur la théorie des équations aux dérivées partielles et la méthode des approximations successives," Journal de Mathématiques Pures et Appliquées, vol. 6, pp. 145-210, 1890.

[6] W. R. Mann, "Mean value methods in iteration," Proceedings of the American Mathematical Society, vol. 4, pp. 506-510, 1953.

[7] S. Ishikawa, "Fixed points by a new iteration method," Proceedings of the American Mathematical Society, vol. 44, pp. 147-150, 1974.

[8] R. Pant and R. Shukla, "Approximating fixed points of generalized $\alpha$-nonexpansive mappings in Banach spaces," Numerical Functional Analysis and Optimization. An International Journal, vol. 38, no. 2, pp. 248-266, 2017.

[9] D. R. Sahu, J. C. Yao, V. K. Singh, and S. Kumar, "Semilocal convergence analysis of $S$-iteration process of Newton-Kantorovich like in Banach spaces," Journal of Optimization Theory and Applications, vol. 172, no. 1, pp. 102-127, 2017.

[10] R. Suparatulatorn, W. Cholamjiak, and S. Suantai, "A modified S-iteration process for G-nonexpansive mappings in Banach spaces with graphs," Numerical Algorithms, pp. 1-12, 2017.

[11] P. Cholamjiak, A. A. Abdou, and Y. J. Cho, "Proximal point algorithms involving fixed points of nonexpansive mappings in CAT(0) spaces," Fixed Point Theory and Applications, vol. 277, 13 pages, 2015.

[12] M. Verma and K. K. Shukla, "A new accelerated proximal technique for regression with high-dimensional datasets," Knowledge and Information Systems, vol. 53, no. 2, pp. 423-438, 2017.

[13] L.-C. Ceng, Q. H. Ansari, and J.-C. Yao, "Some iterative methods for finding fixed points and for solving constrained convex minimization problems," Nonlinear Analysis. Theory, Methods \& Applications. An International Multidisciplinary Journal, vol. 74, no. 16, pp. 5286-5302, 2011.

[14] R. U. Verma, "Projection methods, algorithms, and a new system of nonlinear variational inequalities," Computers \& Mathematics with Applications. An International Journal, vol. 41, no. 7-8, pp. 1025-1031, 2001.

[15] R. U. Verma, "General convergence analysis for two-step projection methods and applications to variational problems," Applied Mathematics Letters. An International Journal of Rapid Publication, vol. 18, no. 11, pp. 1286-1292, 2005.

[16] S. Saejung and K. Wongchan, "A note on Ceng-Wang-Yao's result [Strong convergence theorems by a relaxed extragradient method for a general system of variational inequalities, Math. Meth. Oper. Res. (2008) 67: 375-390]," Mathematical Methods of Operations Research, vol. 73, no. 2, pp. 153-157, 2011.

[17] S. S. Chang, H. W. Joseph Lee, and C. K. Chan, "Generalized system for relaxed cocoercive variational inequalities in Hilbert spaces," Applied Mathematics Letters. An International Journal of Rapid Publication, vol. 20, no. 3, pp. 329-334, 2007.

[18] Z. Huang and M. A. Noor, "An explicit projection method for a system of nonlinear variational inequalities with different $(\gamma, \mathrm{r})$ cocoercive mappings," Applied Mathematics and Computation, vol. 190, no. 1, pp. 356-361, 2007.

[19] X. Qin, S. M. Kang, and M. Shang, "Generalized system for relaxed cocoercive variational inequalities in Hilbert spaces," Applicable Analysis. An International Journal, vol. 87, no. 4, pp. 421-430, 2008.

[20] R. U. Verma, "On a new system of nonlinear variational inequalities and associated iterative algorithms," Mathematical Sciences Research Hot-Line, vol. 3, no. 8, pp. 65-68, 1999.
[21] R. U. Verma, "Generalized class of partial relaxed monotonicity and its connections," Nonlinear Implicit Variational Inequalities, vol. 7, pp. 155-164, 2004.

[22] R. U. Verma, "Generalized system for relaxed cocoercive variational inequalities and projection methods," Journal of Optimization Theory and Applications, vol. 121, no. 1, pp. 203-210, 2004.

[23] Y. Hao, X. Qin, and S. M. Kang, "Systems of relaxed cocoercive generalized variational inequalities via nonexpansive mappings," Mathematical Communications, vol. 16, no. 1, pp. 179-190, 2011.

[24] Q. Dong and D. Jiang, "Solve the split equality problem by a projection algorithm with inertial effects," The Journal of Nonlinear Sciences and Applications, vol. 10, no. 03, pp. 12441251, 2017.

[25] J. H. Zhu, S.-S. Chang, and M. Liu, "Viscosity approximation methods for hierarchical optimization problems of multivalued nonexpansive mappings in CAT(0) spaces," Journal of Nonlinear Science and Applications, vol. 9, no. 10, pp. 5521-5535, 2016.

[26] D. R. Sahu, "Altering points and applications," Nonlinear Studies. The International Journal, vol. 21, no. 2, pp. 349-365, 2014.

[27] A. Latif, D. R. Sahu, and Q. H. Ansari, "Variable KM-like algorithms for fixed point problems and split feasibility problems," Fixed Point Theory and Applications, 2014:211, 20 pages, 2014.

[28] R. P. Agarwal, D. O'Regan, and D. R. Sahu, Fixed point theory for Lipschitzian-type mappings with applications, vol. 6 of Topological Fixed Point Theory and Its Applications, Springer, New York, 2009.

[29] I. Yamada, “The hybrid steepest descent method for the variational inequality problem over the intersection of fixed point sets of nonexpansive mappings," in Inherently parallel algorithms in feasibility and optimization and their applications (Haifa, 2000), vol. 8 of Stud. Comput. Math., pp. 473-504, North-Holland, Amsterdam, 2001. 


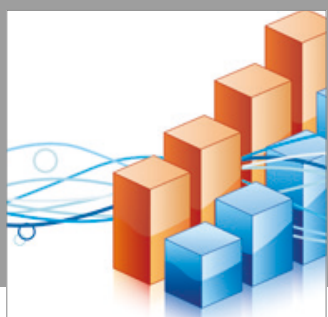

Advances in

Operations Research

vatersals

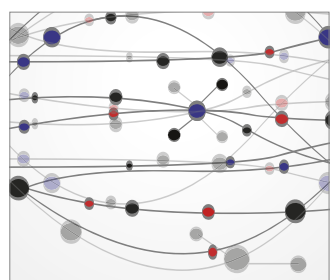

\section{The Scientific} World Journal
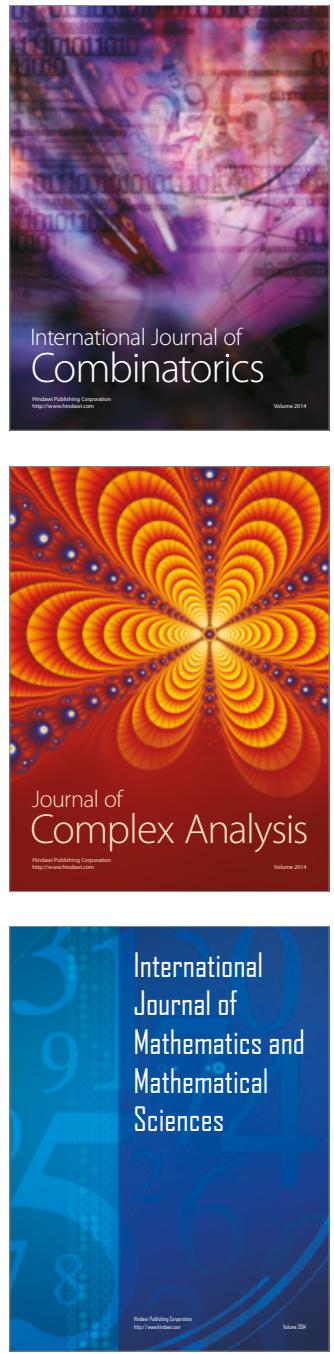
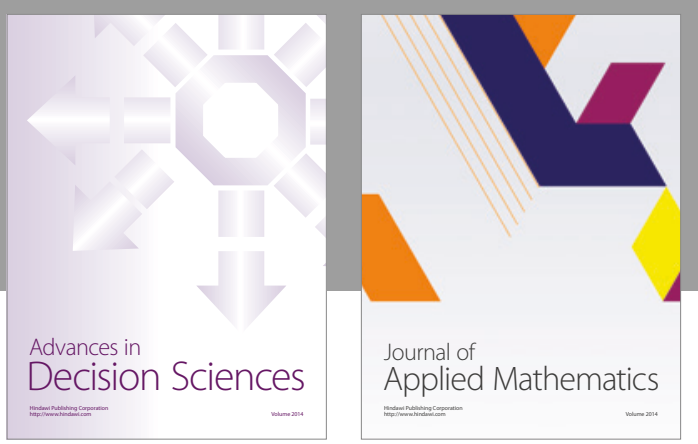

Algebra

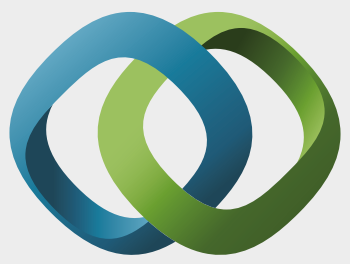

\section{Hindawi}

Submit your manuscripts at

https://www.hindawi.com
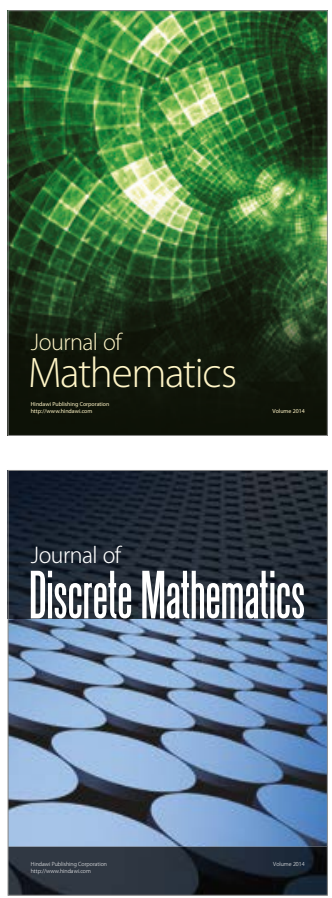

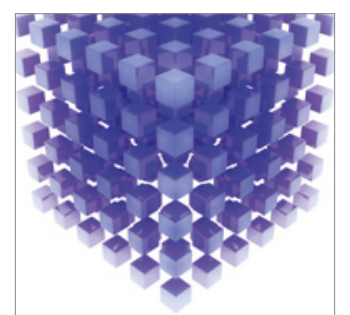

Mathematical Problems in Engineering
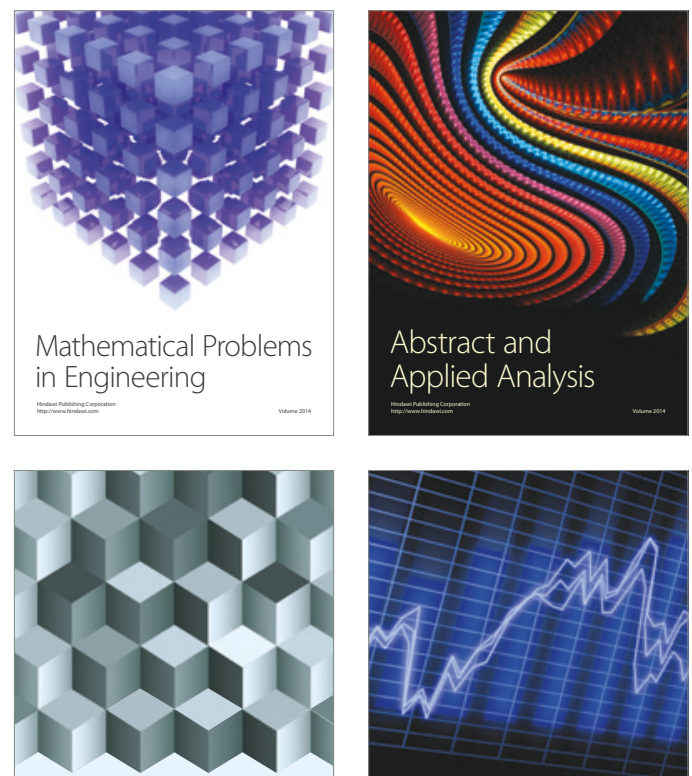

Journal of

Function Spaces

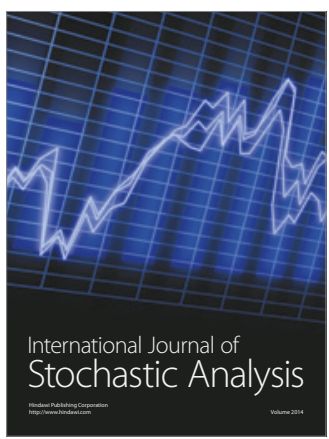

Probability and Statistics
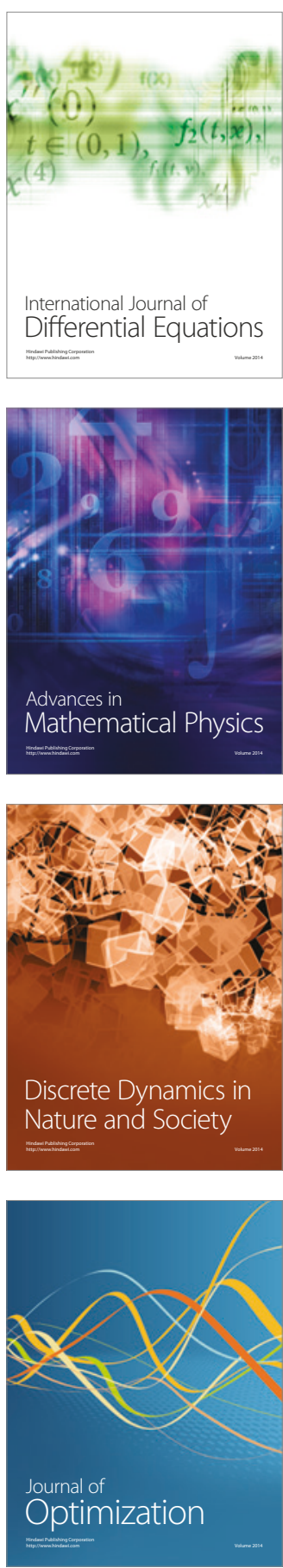